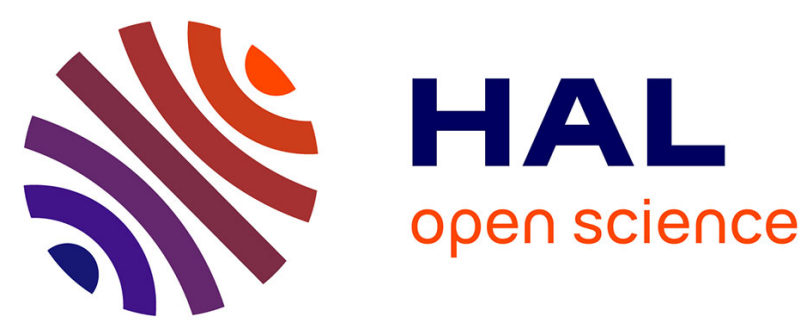

\title{
A wireless, low-power, smart sensor of cardiac activity for clinical remote monitoring
}

Bertrand Massot, Tanguy Risset, Gregory Michelet, Eric Mcadams

\section{To cite this version:}

Bertrand Massot, Tanguy Risset, Gregory Michelet, Eric Mcadams. A wireless, low-power, smart sensor of cardiac activity for clinical remote monitoring. 2015 17th International Conference on Ehealth Networking, Application and Services (HealthCom) (IEEE Healthcom 2015, IEEE, Nov 2015, Boston, United States. pp.487-493. hal-01226432

\section{HAL Id: hal-01226432 \\ https://hal.science/hal-01226432}

Submitted on 9 Nov 2015

HAL is a multi-disciplinary open access archive for the deposit and dissemination of scientific research documents, whether they are published or not. The documents may come from teaching and research institutions in France or abroad, or from public or private research centers.
L'archive ouverte pluridisciplinaire HAL, est destinée au dépôt et à la diffusion de documents scientifiques de niveau recherche, publiés ou non, émanant des établissements d'enseignement et de recherche français ou étrangers, des laboratoires publics ou privés. 


\title{
A wireless, low-power, smart sensor of cardiac activity for clinical remote monitoring
}

\author{
Bertrand Massot, Member, IEEE, Tanguy Risset, Gregory Michelet, Eric McAdams, Senior Member, IEEE
}

\begin{abstract}
This paper presents the development of a wireless wearable sensor for the continuous, long-term monitoring of cardiac activity. Heart rate assessment, as well as heart rate variability parameters are computed in real time directly on the sensor, thus only a few parameters are sent via wireless communication for power saving. Hardware and software methods for heart beat detection and variability calculation are described and preliminary tests for the evaluation of the sensor are presented. With an autonomy of $\mathbf{4 8}$ hours of active measurement and a Bluetooth Low Energy radio technology, this sensor will form a part of a wireless body network for the remote mobile monitoring of vital signals in clinical applications requiring automated collection of health data from multiple patients.
\end{abstract}

Keywords-Wearable sensors, Body sensors network, Biomedical electronics, Biomedical signal processing, Heart rate, Heart rate variability, Autonomic nervous system activity.

\section{INTRODUCTION}

$\mathbf{M}$ OBILE-Health (mHealth) and personal-Health (pHealth) aim to improve the prevention, diagnosis and treatment of diseases, while ensuring patient safety, autonomy, and thus avoiding untimely hospitalization. This in turn will result in more effective healthcare delivery, both financially and therapeutically [1]. The continuous and long-term monitoring of an individual's vital signs enables a real-time and more relevant diagnosis, in order to set up appropriate preventive measures, and to undertake rapid remedial action in the case of early detection of symptoms; as, for example, the latent anticipation of sudden cardiac arrest (424000 annual out-of-hospital cardiac arrests, with an overall survival rate of only $5.2 \%$ [2]) by evaluating the risks of cardiovascular disease and by detecting any cardiac abnormalities. A promising solution is the development of wearable systems which assess relevant indicators enabling a direct, on-body cardiac diagnosis. Several research projects' results in this area have highlighted a range of various technical challenges that must be overcome [3], [4].

The achievement of a suitable device for continuous, longterm monitoring of heart rate activity will enable the detection of cardiac abnormalities in the electrocardiogram signal (ECG), for example to prevent ventricular fibrillation (VF), and to monitor the instantaneous heart rate (HR), from which can

\footnotetext{
Manuscript first submitted 04 Mar 2015.

This work was supported by INSA Lyon under grant RECAMED BQR2015.

B. Massot, G. Michelet and E. McAdams are with the Biomedical Sensors Group, University of Lyon, Lyon Institute of Nanotechnology INL-UMR5270, CNRS, INSA Lyon, Villeurbanne, F-69621, France e-mail: bertrand.massot@insa-lyon.fr

T. Risset is with the CITI Lab, University of Lyon, Inria, INSA-Lyon, Villeurbanne, F-69621, France
}

be derived several parameters regarding heart rate variability (HRV). HRV provides information on autonomic nervous system (ANS) activity, a relevant indicator for several pathologies [5] and more generally on an individual's stress and arousal.

New wearable devices for the monitoring of heart rate activity can exploit the benefits of recent technological advances in electronics and wireless communication systems in order to overcome the challenges previously cited. Prototypes developed in laboratories already show really promising results in terms of wearability, robustness and autonomy, as for example the wearable patch developed at the Holst Centre which benefits from both elaborated hardware [6] and software [7], and more recently from a new kind of dry electrode for comfortable measurements [8]. There are already commercially available products for personal monitoring of one's own cardiac rhythm, but they are mainly aimed at well-being and fitness applications rather than being suitable for medical prevention and diagnosis. Most of these systems suffers from a lack of accuracy, depending on the sensing method used (for example, plethysmography which appears to be still questionable for instantaneous HR and short-term HRV and also suffers from many motion artefacts in ambulatory conditions [9]), and on the calculation methods (for example filtering HR due to motion artefacts induces distortion in frequency content of subsequent HRV parameters).

Therefore several research projects currently focus on improving sensing methods and develop promising tools that can be integrated in future embedded devices, for example the use of flexible capacitive electrodes [10] to minimize motion artefacts, which also avoid skin irritation issues associated with the adhesive of disposable electrodes or the large impedance mismatches encountered when using dry electrodes. Other projects address the challenge of reducing the energy consumption of critical parts of the devices, for example through the use of an integrated, ultra-low power and robust analog front-end for ECG measurement [11] [12]. On the other hand, several research projects since the early 2000's have attempted to develop WBANs for health monitoring; mostly focusing on the development of an infrastructure optimized for reducing power consumption while increasing the reliability of the communication between sensors and remote devices, and also on data security (for complete reviews between 2009 and 2013 see for example [13]-[16], and more recently [17], [18] in 2014 and 2015). Given the panel of technologies and architectures described in the literature, surprisingly only a few of the presented devices progress to a final level of fabrication and approval to make them suitable for clinical trials. This is a critical step in the development of medical applications which could benefit from these novel devices. It has been reported 
that medical indicators defined under laboratory conditions prove to be insufficient, even irrelevant, under the monitoring conditions within more complex environments. This is due to the numerous factors which can adversely influence the parameters calculated, and thus lead to the definition of novel parameters depending on the application or study [19].

In this paper, we present a novel wearable system for continuous long-term monitoring of cardiac activity, in order to improve analysis of daily heart rhythms during in-situ experiments. This system is developed within the frame of the REC@MED project, which aims to develop a wireless body area network (WBAN) for a continuous health monitoring of the individual, at home and on the move. This system includes a new smart sensor, termed the REC Heart Activity monitoring system, for the measurement of ECG and for the real-time calculation of cardiac activity indicators (HR, HRV). The sensor involves a wireless connection to an application embedded in a smartphone, which collect data from the different sensors composing the REC@MED network. We present the materials and methods used to embed, within a programmable and integrated electronic architecture, the direct and real-time calculation of indicators for pertinent analysis of cardiac activity. The underlying hypothesis to be evaluated with this device is the energy saved by real-time processing of the ECG rather than its full transmission over the air, which results in the use of a smaller battery and thus reduces the size of the wearable device. We therefore aimed at combining a robust measurement of the ECG with the accurate onboard calculation of HR and HRV parameters so they can be used for research and medical applications, within a tiny, long autonomy device to enable the daily use of the system.

\section{Materials And Methods}

The REC Heart Activity device is an ambulatory system for the evaluation of electrical heart activity (electrocardiogram, heart rate and heart rate variability). The device comprises a

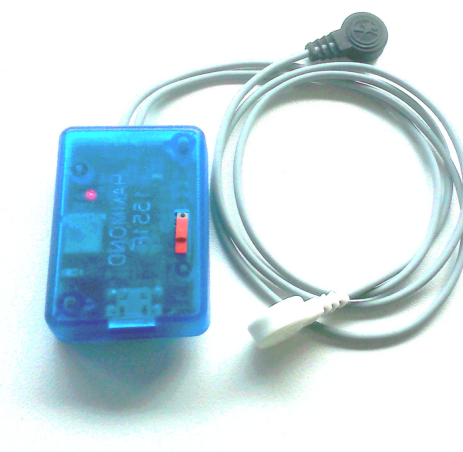

(a)

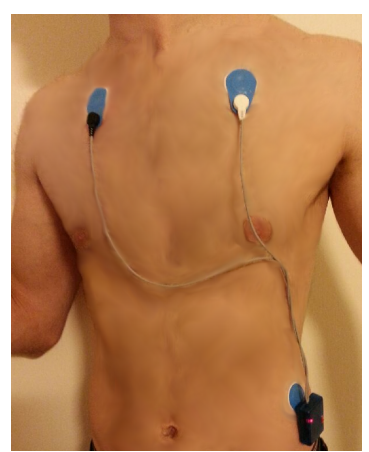

(b)
Fig. 1. Device overview (a) and placement on the body (b)

small electronic board and a $300 \mathrm{mAh}$ Lithium-Ion battery encapsulated in a single $50 \mathrm{~mm} \times 35 \mathrm{~mm} \times 15 \mathrm{~mm}$ plastic enclosure with an overall weight of $35 \mathrm{~g}$ (Fig. 1a). It is connected to three disposable, body-worn ECG electrodes, in a Lead II configuration by means of snap connectors: two are connected through flexible wires, and one directly mounted under the enclosure. This reduced form factor, and the "ondevice" snap connection, enable the discreet, non-intrusive wearing of the device directly on the chest (shown on Fig. 1b). For a simple operation of the system, the device has a single power switch with an associated LED indicating the power state, and provides a universal micro-USB connector so that the battery can be charged with any common mobile device's wall charger or from a computer-hosted USB port. Two additional LEDs indicate a connection to the USB connector and the charging state of the battery.

\section{A. System hardware}

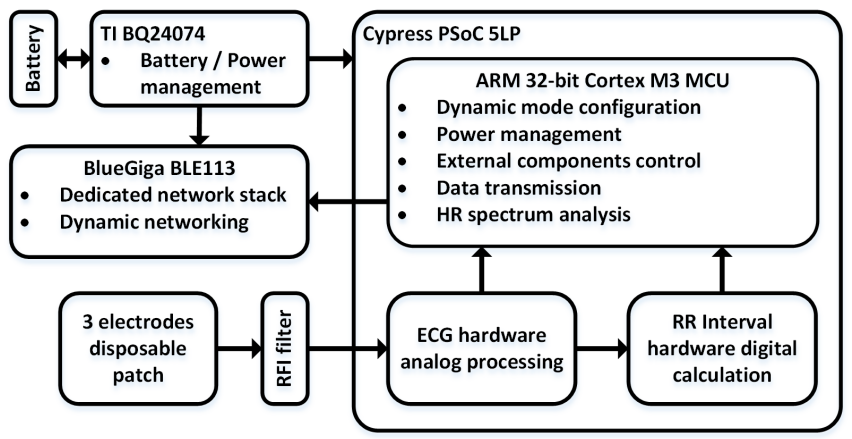

Fig. 2. Electronic architecture overview of the system

The electronic architecture of the board (Fig. 2) is based on a PSoC 5LP (Cypress Semiconductor). This mixed-signal Programmable System-on-Chip consists of a Cortex M3 ARM MCU and includes analog programmable functions and Programmable Logic Device (PLD) based functions. This component can thus carry out all the steps from the conditioning of the ECG signal to the transmission of high level heart activity indicators, including signal processing, analog to digital conversion, hardware heart rate detection, and software heart rate variability calculation. All these functions are integrated within a low-power, single chip with a highly reduced size $(8 \mathrm{~mm} \times 8 \mathrm{~mm})$. Wireless communication is achieved by a BLE113 Bluetooth Smart module (Bluegiga Technologies) which provides Bluetooth 4.0 (Bluetooth Low Energy) radio, software stack, and a programmable GATT-based profile. The device also embeds an integrated Lithium-Ion charger BQ24074 (Texas Instruments) which provides autonomous power-path management for battery charge and system power.

Except for the passive analog filters, the whole ECG conditioning chain is integrated into the PSoC's programmable analog functions. The ECG conditioning chain includes an instrument amplifier connected to a differential, successive approximation, analog-to-digital converter (ADC) which samples the ECG with a 12-bit resolution and a 8192 sps sampling rate. A "Right-Leg Driven" circuit is implemented to provide additional active common-mode noise rejection [20]. Similarly, the hardware implementation of the HR detection is fully integrated into the PSoC's PLD-based components. The method used for calculating the R-R interval is based 
on the method described by Pan and Tompkins [21]: using a Direct Memory Access (DMA) channel, the ADC data goes through a digital filter block (DFB) which acts as an ultralow power digital signal processor. The DFB includes double buffering for the ECG signal to calculate a smoothed derivative which is then squared, and a moving-average filter is applied on the final signal. The local maximum detector is built using PLD components: a peak is detected when both the polarity of the derivative is changing from positive to negative and the signal is above an adaptive threshold. The adaptive threshold, $T$, is dynamically computed within the PLD components using exponential smoothing (Eq. 1), where $\beta$ is the amplitude ratio of the current peak detected $P$ and $\alpha$ is the smoothing factor of the filter.

$$
T_{n+1}=\alpha \cdot \beta \cdot P_{n}+(1-\alpha) \cdot T_{n}
$$

This method provides dynamic and automatic adaptation of the threshold as the amplitude of the ECG signal may vary between individuals and also over time. When a peak is detected, the internal register of a counter is copied directly into the MCU memory and an interrupt is triggered.

\section{B. Embedded MCU Software}

1) Software flowchart: The hardware chain previously described and the software implemented in the MCU are designed to provide an optimized power management of the system. Most of the time, the MCU is put into an "idle" state, and it can receive hardware "wake-up" events to achieve one of the following actions: enable or disable the ECG acquisition and HR detection chains, calculate HRV parameters, and update data of the GATT server into the BLE chip. During active periods, the MCU operates at its minimal clock rate (3 MHz).

2) Calculation of $H R V$ parameters: HRV parameters are calculated according to the recommendations provided by the joint Task Force of the European Society of Cardiology and the North American Society of Pacing and Electrophysiology [5]. The calculations are based on HR segments with a length of $5 \mathrm{~min}$, which is recommended for the calculation of shortterm parameters. Thus the MCU buffers HR values until their sum equals $300 \mathrm{~s}$, and then triggers a sequential calculation of the parameters in Table I. The frequency-domain parameters

TABLE I. DESCRIPTION OF SHORT-TERM HRV PARAMETERS

\begin{tabular}{|c|c|c|c|}
\hline Variable & Unit & Domain & Description \\
\hline SDNN & $\mathrm{ms}$ & Time & Standard deviation of all R-R intervals \\
\hline RMSSD & $\mathrm{ms}$ & Time & $\begin{array}{l}\text { Quadratic mean of differences between succes- } \\
\text { sive R-R intervals }\end{array}$ \\
\hline $\mathrm{LF} / \mathrm{HF}$ & n.u. & Frequency & $\begin{array}{l}\text { Ratio between low-frequency }(0.04 \text { to } \\
0.15 \mathrm{~Hz}) \text { and high-frequency }(0.15 \text { to } \\
0.4 \mathrm{~Hz}) \text { components of the PSD of all R-R } \\
\text { intervals }\end{array}$ \\
\hline LF norm & $\%$ & Frequency & $\begin{array}{l}\text { Ratio (expressed as a percentage) between low- } \\
\text { frequency components, and the sum of low- } \\
\text { and high-frequency components of the PSD, i.e. } \\
\text { LF/(LF+HF) }\end{array}$ \\
\hline
\end{tabular}

require an evaluation of the power spectrum density (PSD) of the HR signal prior to their calculation, however the afore mentioned Task Force is rather unclear on a preferential method to evaluate the PSD. In this application, the method of evaluating the PSD is critical due to the embedded electronic architecture used in the sensor, which provides limited resources in performance and time. As R-R intervals vary in time, the HR signal is composed of unevenly sampled values; thus a traditional approach for spectral analysis consists of a combination of (i) an interpolation, in order to recover an evenly sampled signal, and (ii) a subsequent Fast Fourier Transform (FFT) to obtain the PSD. However this approach, depending on the method of interpolation, the sampling rate and the number of points, is known to introduce distortion in the high-frequency domain where re-sampling acts as a low-pass filter, leading to an overestimation of HRV parameters [22]. Another approach for spectral analysis of an unevenly sampled signal is the least square analysis, commonly termed the Lomb-Scargle periodogram, which provides (in a normalised form), the estimated power $P$ of the angular frequency component $\omega$. This method, originally proposed by Lomb [23] and further elaborated by Scargle [24], was proposed as a surrogate for HRV calculations for the first time by Shin et al. [25] in 1994 (to the best of authors' knowledge). This method provides better accuracy and lower noise levels in the estimation of the density power spectrum, but unfortunately it also has the major drawback of involving much more calculation complexity, and thus CPU time consumption, even when optimized with classical trigonometric recurrences. Press and Rybicki [26] have proposed a much faster computation of this parameter by combining the accuracy of the periodogram and the efficiency of FFT, resulting in an algorithm which is as fast as two FFT calculations. The raw algorithm and several settings of its fast implementation have been compared, and a performance comparison in accuracy and gain of time is presented in the Results section.

\section{Embedded Radio Software}

The BLE113 component is programmed with the standard tool suite provided by BlueGiga, so that the complete Bluetooth 4.0 software stack is already included in the firmware. A custom GATT-server has been designed and its attributes are illustrated on Table II. This server implements the standard

TABLE II. BLE GATT SERVER INCLUDING STANDARD HEART HEART PROFILE AND ADDITIONAL CHARACTERISTICS

\begin{tabular}{|c|c|c|}
\hline Type & Description & Std. UUID \\
\hline \hline Service & Generic Access Profile & 1800 \\
Characteristic & Device Name & $2 \mathrm{a} 00$ \\
Characteristic & Appearance & $2 \mathrm{a} 01$ \\
\hline Service & Device Information & $180 \mathrm{a}$ \\
Characteristic & Manufacturer Name String & $2 \mathrm{a} 29$ \\
Characteristic & Model Number String & $2 \mathrm{a} 24$ \\
\hline Service & Heart Rate Service & $180 \mathrm{~d}$ \\
Characteristic & HR Measurement & $2 \mathrm{a} 37$ \\
Characteristic & Body Sensor Location & $2 \mathrm{a} 38$ \\
Characteristic & HRV Measurement & - \\
\hline Service & Battery Service & $180 \mathrm{f}$ \\
Characteristic & Battery Level & $2 \mathrm{a} 19$ \\
\hline
\end{tabular}


Heart Rate Profile as defined by the Bluetooth 4 specifications with an additional characteristic for HRV parameters.

\section{Android mobile interface}

An application compatible with the versions 4.3 and higher of the Android operating system (for Bluetooth 4 support) has been developed in order to log onto the user's phone the data provided by the sensor. The application is divided into three main software interfaces illustrated on Fig. 3. The first

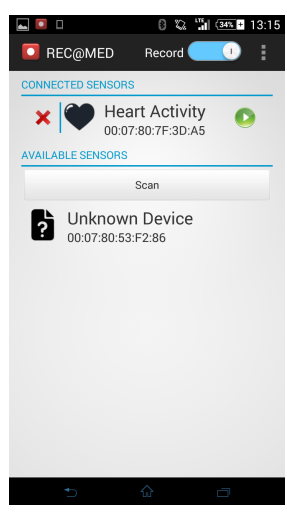

(a)

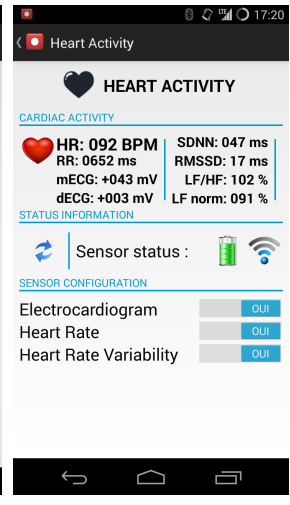

(b)

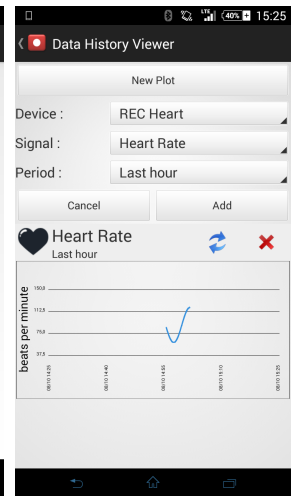

(c)
Fig. 3. Preview of the Android application including the WBAN manager interface (a), the sensor configuration interface (b) and the signal viewer interface (c).

interface manages connections to a range of supported sensors in the REC@MED project including the REC Heart Activity device. This interface controls a background service which can maintain connections to the sensors even when the smartphone is in an idle state, to prevent the OS closing the application when the screen is off for battery saving. When recording is activated, every event or data received from any sensor of the network is logged into a unique user's SQLite database on the smartphone. In this way, the service can record data onto the database regardless of the source or the type of the data received. The second interface is dedicated to the configuration of the connected sensors. For each supported sensor, a specific GUI provides real-time information concerning the signals sent and enables the configuration of the notifications from the GATT-server of the sensor. The third interface enables the previewing of the data recorded into the database within four pre-defined periods of time: the data recorded during the last hour, day, week or month from the current time of viewing.

\section{E. Data security}

The whole system benefits from the platform-tools provided to enhance data security during transmission and within the records. For instance, the Bluetooth 4.0 connection between the Android application and the sensors uses an AES-128 with CCM encryption engine. To record the data into a SQLite database, the Android application uses the SQLCipher library to encrypt the database with a 256-bit AES algorithm in CBC mode.

\section{F. Evaluation of the system}

Preliminary tests in laboratory and also under real life situations were carried out to evaluate (i) the accuracy HR and HRV parameter calculations, (ii) the robustness of R-peaks detection under real life conditions and (iii) the usability for long-term experiments (autonomy, ease of use, etc).

The system was evaluated on a workbench with a test ECG signal, generated by an Agilent 33220A. This device provides an arbitrary waveform generator, including a pre-built cardiac signal. Period and amplitude of the signal can be tuned, and the device also provides amplitude modulation, with adjustable depth to simulate the variations of amplitude due to the shifting skin/electrode impedance in time, and frequency modulation to simulate the natural variability of the human heart rate. Results of the tests are presented in sections III-A and III-B.

Preliminary tests were conducted under real-life conditions, where the sensor was attached to electrodes (Blue Sensor, Ambu) for ECG measurements. The system was worn over working days to evaluate comfortableness, signal robustness, and wireless connection reliability. The measurements were also compared simultaneously to two commercially available devices for daily heart rate monitoring, a textile electrodebased monitoring device (WWS) and a new wrist based monitoring device using photoplethysmography (VVW). Results of the tests are presented in section III-C.

\section{RESULTS}

\section{A. Accuracy of HR assessment using a generated ECG signal}

Accuracy of the detection of R-R intervals was evaluated using an Agilent 33220A as the ECG generator. The error in $\mathrm{R}-\mathrm{R}$ interval measurement was calculated as the difference between the period set on the generator and the period detected by the sensor with a precision of $0.98 \mathrm{~ms}$, as the sensor sends values of R-R intervals using a binary clock with an accuracy of $1024 \mathrm{~Hz}$. The sample used contained $1000 \mathrm{R}-\mathrm{R}$ intervals whose lengths were linearly varied from $400 \mathrm{~ms}$ (150 BPM) up to $1200 \mathrm{~ms}$ (50 BPM) which is representative of most common heart rates. The mean value of all differences was $-0.105 \mathrm{~ms}$ and the standard deviation was $1.027 \mathrm{~ms}$ over all the range of $\mathrm{R}-\mathrm{R}$ intervals. For the calculated HR, a difference of $1.027 \mathrm{~ms}$ results in an error of $0.1 \mathrm{BPM}$ when $\mathrm{HR}$ is $60 \mathrm{BPM}$, and 0.4 $\mathrm{BPM}$ when HR is $120 \mathrm{BPM}$.

\section{B. Accuracy of PSD estimation using the fast implementation of Lomb-Scargle periodogram}

The different methods for PSD evaluation were compared by applying a frequency modulation (FM) to the ECG signal generated by the Agilent 33220A. The base HR was set at $1.25 \mathrm{~Hz}$ (75 BPM); and the modulating signal had a frequency of $0.05 \mathrm{~Hz}$ with an amplitude of $0.2 \mathrm{~Hz}$ and a triangular shape. The use of a triangular shape as a modulating signal has the advantage of inducing harmonics in the PSD at multiple frequencies of the fundamental frequency to populate the PSD with non-null frequency components all over the range of interest $(0.015 \mathrm{~Hz}$ to $0.4 \mathrm{~Hz})$. Additionally, the PSD is estimated 
over R-R periods and not over the linearly varied HR. The nonlinear relationship between period and frequency will induce additional even harmonics into the spectrum of a triangular shape, which originally contains only odd harmonics.

The fast implementation of Lomb-Scargle periodogram relies on the computation of two FFTs of a vector containing R-R interval values which are extirpolated by a factor (MACC) that determines the size of the vector, and thus the calculation time of FFT. Three different values of the MACC parameter were tested to evaluate the quality of the PSD versus calculation time for HR segments having a duration of $5 \mathrm{~min}$. Table III presents the calculation times for each factor value, and Fig. 4 illustrates the corresponding quality of PSD. All methods

TABLE III. CALCULATION TIME FOR EACH METHOD OF PSD ESTIMATION OF R-R INTERVALS FOR 5 min LONG SEGMENTS.

\begin{tabular}{|c|c|}
\hline Type & Time \\
\hline Normalized LP & $>60 \mathrm{~s}$ \\
Fast LP (MACC $=4)$ & $4 \mathrm{~s}$ \\
Fast LP (MACC $=2)$ & $2 \mathrm{~s}$ \\
Fast LP (MACC $=1)$ & $1 \mathrm{~s}$ \\
\hline
\end{tabular}

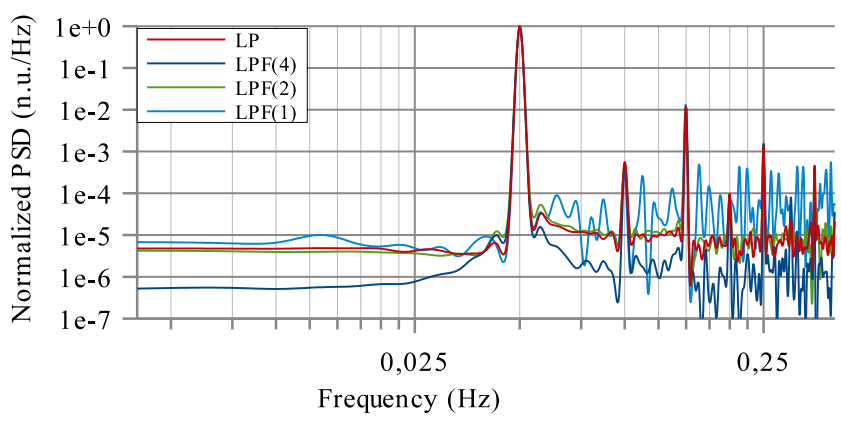

Fig. 4. Comparison of raw implementation of Lomb-Scargle (LP) and its fast implementation $\operatorname{LPF}(1), \operatorname{LPF}(2)$ and $\operatorname{LPF}(4)$, for a $0.05 \mathrm{~Hz}$ triangular modulation of HR.

produced an estimation of PSD with equivalent power for the different harmonic components of the modulating signal. The results clearly show that $\operatorname{LPF}(2)$ (fast implementation of the Lomb-Scargle periodogram with a MACC factor of 2) provides the best quality/time compromise. The estimated PSD with this method is the closest to the original while having a reasonably short calculation time, allowing a quick return of the MCU in a sleep state; besides, the fastest method, LPF(1), produces non negligible noise in the upper frequencies (Fig. 4).

\section{Robustness of R-Peak detection under real life conditions}

As a preliminary test of a daily use of the sensor as a continuous monitoring system for heart rate variability, a volunteer from the research group accepted to wear the device during working days to test signal robustness and connection reliability to the smartphone application. Additionally a comparison with two other commercially available devices was made during one day. Collected data have shown that the communication reliability on the smartphone has to be strengthen as the Android operating system spontaneously killed the application process to free up memory (at an average rate of twice a day). In the future, a scheduled service will be implemented to check regularly if the process is running when the user chooses to maintain a continuous, long-term recording on the phone. An example of a 28 hours continuous record of $\mathrm{R}-\mathrm{R}$ intervals on the phone is illustrated on Fig. 5 .

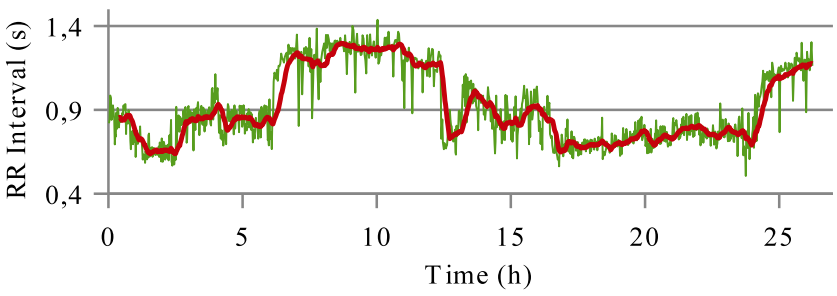

Fig. 5. Continuous record of R-R intervals starting at $6.00 \mathrm{pm}$ and stopping at $10.00 \mathrm{pm}$ the day after (instantaneous intervals and a 5 minutes moving average are colored in green in red respectively).

On the other hand, the data recorded by the device (REC) were of at least equivalent accuracy compared to the WWS, as the difference in true positive detection values was due to the difference of sampling rate (WWS has a lower sampling rate of $250 \mathrm{sps}$ which can lead to differences up to $4 \mathrm{~ms}$, i.e. the range of measurement error). Additionally, during the entire day period of testing, the REC Heart Activity has shown only one false positive detection while the specificity indicator of the WWS decreased with the level of physical activity and the drying of textile electrodes which needed periodic moistening (about every four hours of measurement). These false detections due to movement artifacts and higher impedance of skin/electrode contact are illustrated on Fig. 6 and 7 for resting and activity periods respectively. Beside, while the wrist-worn

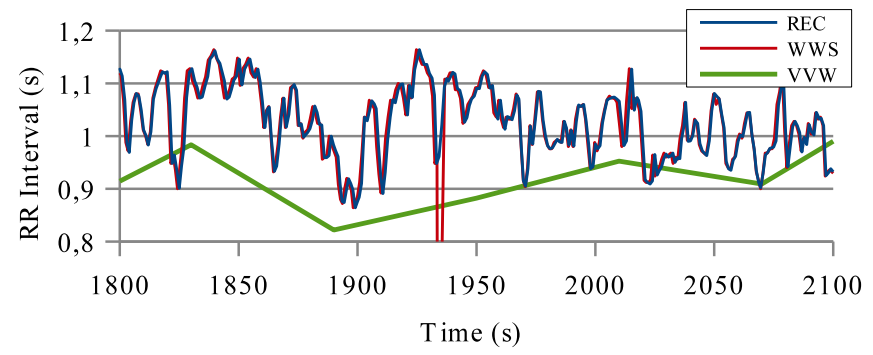

Fig. 6. Comparison of simultaneous R-R intervals measured by three different wearable systems during rest period.

device (VVW) features a real-time monitoring of HR on an integrated screen, it was not possible to record these data and only one-minute averaged values could be recovered from a $\log$ file. Moreover, either during resting and physical activity periods, the device suffers from a strong lack of accuracy, showing that it is not intended for a medical usage.

\section{Power consumption and autonomy of the system}

The main state of the system, during normal use of the sensor, is an active state during which ECG is continuously 


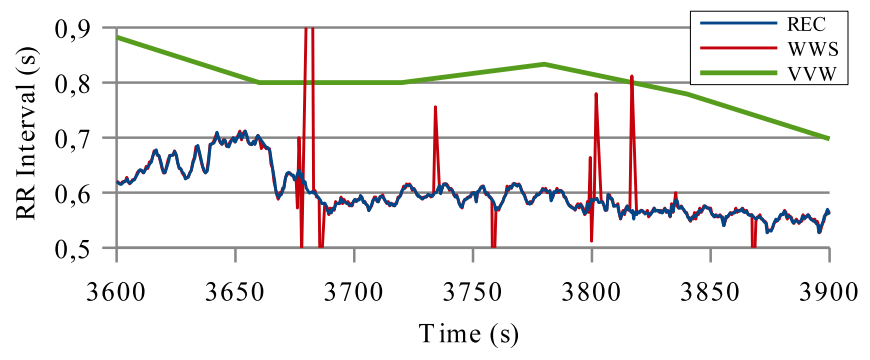

Fig. 7. Comparison of simultaneous R-R intervals measured by three different wearable systems during physical activity.

sampled for HR detection and HRV calculations. While in this state, the average consumption of the system is lower than $5 \mathrm{~mA}$ drawn from a $3.7 \mathrm{~V}, 300 \mathrm{mAh}$ Lithium-Polymer battery. Thus the current system provides more than 48 hours of continuous calculation of heart rate variability when the system is worn. The major part of this current requirement is due to the non-specific analog frontend for ECG measurement within the PSoC. This is the major drawback of using this type of technology instead of developing an optimized and dedicated frontend [6], but it has the advantage of reducing the number of components, and thus the size of the overall device.

\section{DISCUSSION}

\section{A. R-peak detection and PSD estimation methods}

Several advanced processing methods have been developed ensuring high robustness for the detection of R-peak on the ECG, for example by detecting the peaks in other domains than the time-domain (Hilbert transform [27], or Wavelet transform [28]), or by using trained algorithms [29]. However Pan and Tompkins' method implemented in the REC Heart Activity device resulted in highly accurate and reliable data during the preliminary tests conducted within the research group, thanks to a robust measurement of ECG signals. This choice of implementation was guided by the opportunity of using an ultra low-power DSP integrated within the Systemon-Chip, as it freed the MCU from intensive and frequent calculations, which could not been avoided with the use of other methods on this particular architecture. Regarding the implementation of an embedded, real-time algorithm for the estimation of power spectral density of R-R periods, the LombScargle periodogram was selected for its overall accuracy in the estimation of low and high frequency ranges. Primarily this method induces less distortion in the upper frequency range compared to the traditional successive interpolation and Fast Fourier Transform of the tachogram, while being more robust to missing data. As previously shown, the original implementation of the periodogram requires long periods of MCU calculation, but an affordable compromise has been proposed : although this calculation lasts about 2 seconds, this event occurs only once every 5 minutes of data acquisition, corresponding to an additional duty cycle of $0.7 \%$ of MCU time consumption. However both approaches remain sensitive to non-stationary heart rate segments, which can lead to erroneous interpretation of HRV parameters in so-called "non stationary" environments, for example during social interactions. Thus other methods will be investigated in the future to improve the sensor, as the software implementation of PSD estimation enables one to change the method used, with for example Robust Detection Period method [30].

\section{B. Daily use of the device for a long term monitoring}

The device is primarily intended to be used in research experiments under real-life conditions which specifically require a non-invasive and embedded device to enable ambulatory measurements. As presented in the Results section, this device has a full autonomy of two days without charging the battery, and uses high-performance "wet" -gel electrodes to assess ECG for a better signal reliability during physical exertion. These are two potential limitations to extending the use of this device, as a daily monitoring system, to very long term monitoring involving weeks or months of measurements. However, a few additional materials and methods could be implemented to enable a longer use of the device. Firstly the actual use of a low capacity battery within the device results in faster charging. The embedded charger has been set to charge the battery with a $300 \mathrm{~mA}$ current, so an average duration of one hour is needed to charge the entire battery from a full discharged former state. If a specific time interval of half an hour can be used every-day for a partial charging of the battery, this will ensure an unlimited use of the sensor in time; typically, this operation can be handled during the time spent, for example, in the bathroom where the device has to be removed. Regarding the electrodes, the use of adhesive wet electrodes is prohibited for very long term measurement, as the quality of the signal will decrease over time and the adhesive part will result in skin irritation problems. Thus the use of highperformance dry electrodes, conductive or capacitive-coupled, is recommended, and could be built into a belt or weaved into a specific textile, as for example, the promising EPDM electrodes recently developed at the Holst Centre [8].

\section{CONCLUSION}

In this paper we have presented a smart, ready-to-use, sensor for the continuous and long term monitoring of heart rate and heart rate variability. The sensor implements an embedded, real-time calculation of $\mathrm{HR}$ and $\mathrm{HRV}$ parameters in order to save power consumption and reduce the amount of data transmitted in wireless communications. This portable system is also adaptable to different placements and different kinds of electrodes as it is directly clipped onto one of the electrodes, thus enabling a more discrete and comfortable wearing of the system.

This paper has shown that, despite the fact that very few systems are currently commercially available for both comfortable and high quality assessment of HRV, technology advances already enable the design of smart physiological sensors which are convenient for a medical usage by clinicians. More generally, the REC@MED project aims to propose a global hardware architecture, with sensors newly developed 
for cardiac, electrodermal and physical activity monitoring together, as well as a software platform on smartphone for collecting, storing and passing on data securely. This WBAN is proposed as a solution for the increasing clinical need of automated collection of health data from multiple patients, both inside and outside of a medical enviroment (hospital or nursing home).

\section{REFERENCES}

[1] C. Van Hoof and J. Penders, "Addressing the healthcare cost dilemma by managing health instead of managing illness: an opportunity for wearable wireless sensors," in Proceedings of the Conference on Design, Automation and Test in Europe. EDA Consortium, 2013, pp. 15371539.

[2] A. S. Go, D. Mozaffarian, V. L. Roger, E. J. Benjamin, J. D. Berry, M. J. Blaha, S. Dai, E. S. Ford, C. S. Fox, S. Franco et al., "Heart disease and stroke statistics-2014 update: a report from the american heart association." Circulation, vol. 129, no. 3, p. e28, 2014.

[3] E. McAdams, A. Krupaviciute, C. Gehin, E. Grenier, B. Massot, A. Dittmar, P. Rubel, and J. Fayn, "Wearable sensor systems: The challenges," in Engineering in Medicine and Biology Society,EMBC, 2011 Annual International Conference of the IEEE, 30 2011-sept. 3 2011, pp. $3648-3651$.

[4] B. Massot, N. Noury, C. Gehin, and E. McAdams, "On designing an ubiquitous sensor network for health monitoring," in 2013 IEEE 15th International Conference on e-Health Networking, Applications and Services (Healthcom) (IEEE Healthcom 2013), Lisbon, Portugal, Oct. 2013.

[5] M. Malik, J. T. Bigger, A. J. Camm, R. E. Kleiger, A. Malliani, A. J. Moss, and P. J. Schwartz, "Heart rate variability standards of measurement, physiological interpretation, and clinical use," European heart journal, vol. 17, no. 3, pp. 354-381, 1996.

[6] M. Altini, S. Polito, J. Penders, H. Kim, N. Van Helleputte, S. Kim, and F. Yazicioglu, "An ecg patch combining a customized ultra-lowpower ecg soc with bluetooth low energy for long term ambulatory monitoring," in Proceedings of the 2nd Conference on Wireless Health. ACM, 2011, p. 15.

[7] I. Romero, B. Grundlehner, and J. Penders, "Robust beat detector for ambulatory cardiac monitoring," in Engineering in Medicine and Biology Society, 2009. EMBC 2009. Annual International Conference of the IEEE. IEEE, 2009, pp. 950-953.

[8] Y.-H. Chen, M. Op de Beeck, L. Vanderheyden, V. Mihajlovic, B. Grundlehner, and C. Van Hoof, "Comb-shaped polymer-based dry electrodes for eeg/ecg measurements with high user comfort," in Engineering in Medicine and Biology Society (EMBC), 2013 35th Annual International Conference of the IEEE. IEEE, 2013, pp. 551-554.

[9] A. Schäfer and J. Vagedes, "How accurate is pulse rate variability as an estimate of heart rate variability?: A review on studies comparing photoplethysmographic technology with an electrocardiogram," International journal of cardiology, vol. 166, no. 1, pp. 15-29, 2013.

[10] J. S. Lee, J. Heo, W. K. Lee, Y. G. Lim, Y. H. Kim, and K. S. Park, "Flexible capacitive electrodes for minimizing motion artifacts in ambulatory electrocardiograms," Sensors, vol. 14, no. 8, pp. 14732 14743,2014

[11] S. Izumi, K. Yamashita, M. Nakano, T. Konishi, H. Kawaguchi, H. Kimura, K. Marumoto, T. Fuchikami, Y. Fujimori, H. Nakajima, T. Shiga, and M. Yoshimoto, "A 14 a ecg processor with robust heart rate monitor for a wearable healthcare system," in ESSCIRC (ESSCIRC), 2013 Proceedings of the, Sept 2013, pp. 145-148.

[12] M. Khayatzadeh, X. Zhang, J. Tan, W.-S. Liew, and Y. Lian, "A 0.7v 17.4-/spl mu/w 3-lead wireless ecg soc," Biomedical Circuits and Systems, IEEE Transactions on, vol. 7, no. 5, pp. 583-592, Oct 2013.

[13] H. Cao, V. Leung, C. Chow, and H. Chan, "Enabling technologies for wireless body area networks: A survey and outlook," Communications Magazine, IEEE, vol. 47, no. 12, pp. 84-93, Dec 2009.
[14] S. Ullah, P. Khan, N. Ullah, S. Saleem, H. Higgins, and K. S. Kwak, "A Review of Wireless Body Area Networks for Medical Applications," ArXiv e-prints, vol. 2, no. 8, pp. 797-803, Jan. 2010.

[15] A. Pantelopoulos and N. Bourbakis, "A survey on wearable sensorbased systems for health monitoring and prognosis," Systems, Man, and Cybernetics, Part C: Applications and Reviews, IEEE Transactions on, vol. 40, no. 1, pp. 1-12, Jan 2010.

[16] B. Malik and V. Singh, "A survey of research in wban for biomedical and scientific applications," Health and Technology, vol. 3, no. 3, pp. 227-235, 2013. [Online]. Available: http://dx.doi.org/10.1007/s12553013-0056-5

[17] S. Mukhopadhyay, "Wearable sensors for human activity monitoring: A review," Sensors Journal, IEEE, vol. 15, no. 3, pp. 1321-1330, March 2015.

[18] K. Wac and C. Tsiourti, "Ambulatory assessment of affect: Survey of sensor systems for monitoring of autonomic nervous systems activation in emotion," Affective Computing, IEEE Transactions on, vol. 5, no. 3, pp. 251-272, July 2014.

[19] B. Massot, N. Baltenneck, C. Gehin, A. Dittmar, and E. McAdams, "Emosense: An ambulatory device for the assessment of ans activity application in the objective evaluation of stress with the blind," Sensors Journal, IEEE, vol. 12, no. 3, pp. 543 -551, march 2012.

[20] B. B. Winter and J. G. Webster, "Driven-right-leg circuit design," Biomedical Engineering, IEEE Transactions on, vol. 30, no. 1, pp. 6266, 1983.

[21] J. Pan and W. Tompkins, "A real-time qrs detection algorithm." IEEE transactions on bio-medical engineering, vol. 32, no. 3, pp. 230-236, 1985.

[22] G. D. Clifford and L. Tarassenko, "Quantifying errors in spectral estimates of hrv due to beat replacement and resampling," Biomedical Engineering, IEEE Transactions on, vol. 52, no. 4, pp. 630-638, 2005.

[23] N. R. Lomb, "Least-squares frequency analysis of unequally spaced data," Astrophysics and space science, vol. 39, no. 2, pp. 447-462, 1976.

[24] J. D. Scargle, "Studies in astronomical time series analysis. ii-statistical aspects of spectral analysis of unevenly spaced data," The Astrophysical Journal, vol. 263, pp. 835-853, 1982 .

[25] K. Shin, H. Minamitani, S. Onishi, H. Yamazaki, and M. Lee, "The direct power spectral estimation of unevenly sampled cardiac event series," in Engineering in Medicine and Biology Society, 1994. Engineering Advances: New Opportunities for Biomedical Engineers. Proceedings of the 16th Annual International Conference of the IEEE. IEEE, 1994, pp. 1254-1255.

[26] W. H. Press and G. B. Rybicki, "Fast algorithm for spectral analysis of unevenly sampled data," The Astrophysical Journal, vol. 338, pp. 277-280, 1989.

[27] D. Benitez, P. Gaydecki, A. Zaidi, and A. Fitzpatrick, "A new qrs detection algorithm based on the hilbert transform," in Computers in Cardiology 2000. IEEE, 2000, pp. 379-382.

[28] P. Sasikala and R. Wahidabanu, "Robust $r$ peak and qrs detection in electrocardiogram using wavelet transform," International Journal of Advanced Computer Science and Applications-IJACSA, vol. 1, no. 6, pp. 48-53, 2010.

[29] O. Adeluyi and J.-A. Lee, "R-reader: A lightweight algorithm for rapid detection of ecg signal r-peaks," in Engineering and Industries (ICEI), 2011 International Conference on. IEEE, 2011, pp. 1-5.

[30] J. Skotte and J. Kristiansen, "Heart rate variability analysis using robust period detection," BioMedical Engineering OnLine, vol. 13, no. 1, p. 138, 2014. [Online]. Available: http://www.biomedical-engineeringonline.com/content/13/1/138 